\title{
Micafungin compared with caspofungin for the treatment of febrile episodes in neutropenic patients with hematological malignancies: A retrospective study
}

\author{
Sarah Shalhoub MD, Luchen Wang BSc, Arthur Ching BASc, Shahid Husain MD, Coleman Rotstein MD
}

\begin{abstract}
S Shalhoub, L Wang, A Ching, S Husain, C Rotstein. Micafungin compared with caspofungin for the treatment of febrile episodes in neutropenic patients with hematological malignancies: A retrospective study. Can J Infect Dis Med Microbiol 2014;25(6):299-304.
\end{abstract}

BACKGROUND: Invasive fungal infections are associated with morbidity and mortality in neutropenia secondary to hematological malignancies. Empirical antifungal agents are used to reduce their consequences. Caspofungin is the only echinocandin approved for this indication. Micafungin was compared with caspofungin for the treatment of patients with hematological malignancies and prolonged neutropenia.

METHODS: A retrospective cohort study was conducted involving patients who had hematological malignancies with profound neutropenia for a minimum of 10 days, and received empirical micafungin or caspofungin for a minimum of five days, between April 2005 and November 2009. Successful outcome was based on a composite end point: survival for a minimum of seven days following antifungal cessation, successful treatment of baseline fungal infection, absence of adverse events and absence of breakthrough fungal infection. Fungal infections were defined according to revised definitions of invasive fungal disease from the European Organization for Research and Treatment of Cancer/Invasive Fungal Infections Cooperative Group and the National Institute of Allergy and Infectious Diseases Mycoses Study Group (EORTC-MSG) criteria, with modification of the diagnostic imaging criteria.

RESULTS: Micafungin had similar overall success to caspofungin (60.4\% [29 of 48] versus 57.3\% [47 of 82], respectively; $\mathrm{P}=0.729$ ). Survival was higher in the micafungin group compared with the caspofungin group ( $100 \%$ [ 48 of 48 ] versus $89 \%$ [73 of 82 ]; $\mathrm{P}=0.02$ ). No baseline invasive fungal infections were identified in the micafungin group, compared with three proven infections treated successfully with caspofungin (3.7\%; $\mathrm{P}=0.18)$. Three proven breakthrough infections were observed in the micafungin group (three of 48 [27.3\%]) compared with none in the caspofungin group (zero of $82 ; \mathrm{P}=0.02$ ).

CONCLUSION: Micafungin has similar efficacy to caspofungin as empirical antifungal therapy in febrile neutropenic patients with hematological malignancies. Verification of these results in a prospective trial is warranted.

Key Words: Echinocandins; Fungal; Infection; Leukemia; Neutropenia

Tnvasive fungal infections (IFIs) are a major cause of morbidity 1 and mortality in patients with hematological malignancies during prolonged episodes of neutropenia precipitated by chemotherapy (1). To combat this predisposition to IFI in this group of patients, multiple strategies have been used, namely prophylaxis, preemptive and empirical treatments. There are several candidate antifungals available for

\author{
La micafongine comparée à la caspofongine \\ pour traiter la fièvre chez les patients \\ neutropéniques atteints d'un cancer \\ hématologique : une étude rétrospective
}

HISTORIQUE : Les infections fongiques invasives s'associent à la morbidité et à la mortalité en cas de neutropénie secondaire à un cancer hématologique. Les antifongiques empiriques sont utilisés pour en réduire les conséquences. La caspofongine est la seule échinocandine approuvée pour cette indication. La micafongine lui a été comparée pour traiter des patients atteints d'un cancer hématologique et d'une neutropénie prolongée.

MÉTHODOLOGIE : Les chercheurs ont mené une étude de cohorte rétrospective auprès de patients atteints d'un cancer hématologique et d'une neutropénie profonde pendant au moins dix jours et qui avaient reçu de la micafongine ou de la caspofongine empirique pendant au moins cinq jours entre avril 2005 et novembre 2009. Les résultats positifs se fondaient sur un paramètre ultime composite : survie au moins sept jours après l'arrêt de l'antifongique, résolution de l'infection fongique de départ, absence d'effets indésirables et de percée de l'infection fongique. Les infections fongiques étaient définies conformément aux critères des définitions révisées de maladie fongique invasive de l'Organisation européenne de recherche sur le traitement du cancer et l'Invasive Fungal Infections Cooperative Group et du National Institute of Allergy and Infectious Diseases Mycoses Study Group (EORTC-MSG), avec la modification aux critères d'imagerie diagnostique.

RÉSULTATS : La micafongine fonctionnait de manière similaire à la caspofongine (60,4 \% [29 sur 48] plutôt que 57,3 \% [47 sur 82], respectivement; $\mathrm{P}=0,729)$. La survie était plus élevée dans le groupe sous micafongine que sous caspofongine (100 \% [48 sur 48] plutôt que $89 \%$ [73 sur 82$] ; \mathrm{P}=0,02)$. Aucune infection fongique invasive n'a été constatée au départ dans le groupe prenant de la micafongine, mais trois infections démontrées traitées à la caspofongine $(3,7 \% ; \mathrm{P}=0,18)$ l'ont été. Trois percées d'infections démontrées été observées dans le groupe prenant de la micafongine (trois sur 48 [27,3\%]), mais aucune dans celui prenant de la caspofongine (zéro à $82 ; \mathrm{P}=0,02$ ).

CONCLUSION : La micafongine a une efficacité similaire à celle de la caspofongine comme thérapie antifongique empirique chez les patients neutropéniques fébriles atteints d'un cancer hématologique. Il faudra vérifier ces résultats dans un essai prospectif.

use including the polyenes and echinocandins. In empirical antifungal therapy in febrile neutropenic patients, two key properties are desired: efficacy and reduced toxicity. Although amphotericin B and its lipid formulations have been used as empirical treatments for high-risk febrile neutropenic patients, their unfavourable side effect profile is an impediment. Over the past decade, caspofungin, an echinocandin,

Immunocompromised Host Infectious Diseases Service, Division of Infectious Diseases, Department of Medicine, University Health Network,

University of Toronto, Toronto, Ontario

Correspondence and reprints: Dr Coleman Rotstein, University Health Network, University of Toronto, 200 Elizabeth Street, NCSB 11-1212,

Toronto, Ontario M5G 2N2. Telephone 416-340-4800 ext 5667, fax 416-340-5442, e-mail coleman.rotstein@uhn.ca

OPEN $\bigcirc$ ACCESS

This open-access article is distributed under the terms of the Creative Commons Attribution Non-Commercial License (CC BY-NC) (http:// creativecommons.org/licenses/by-nc/4.0/), which permits reuse, distribution and reproduction of the article, provided that the original work is properly cited and the reuse is restricted to noncommercial purposes. For commercial reuse, contact support@pulsus.com 
was found to be noninferior to amphotericin B and liposomal amphotericin B in treatments of invasive candidiasis and persistent fever and neutropenia, respectively $(2,3)$. As a result, the echinocandins were promptly included in recommendations for treatment of invasive candidiasis by the Infectious Diseases Society of America (4). Caspofungin was also approved in empirical antifungal treatment of febrile neutropenic patients $(1,5)$.

There is a paucity of data regarding the use of micafungin as empirical antifungal treatment in febrile neutropenic patients with hematological malignancies. Kubiak et al (6) evaluated the use of micafungin compared with caspofungin as antifungal treatment in persistently febrile neutropenic patients in a sequential retrospective cohort. Similar efficacy was observed in terms of survival, treatment of baseline IFIs (despite a small number) and incidence of breakthrough fungal infections. However, the data were limited by the fact that prolonged neutropenia (an established risk factor for invasive fungal infections, as per European Organization for Research and Treatment of Cancer/Invasive Fungal Infections Cooperative Group and the National Institute of Allergy and Infectious Diseases Mycoses Study Group [EORTC-MSG] criteria and the Infectious Diseases Society of America) was not a prerequisite to include patients in the analysis, especially because IFIs are less likely to develop early during chemotherapy-induced neutropenia $(1,5)$. The other limiting factor was the inclusion of patients who received as few as two doses of caspofungin or micafungin, which may not reflect true efficacy. This may have affected the outcome of the analysis.

Therefore, we conducted the present retrospective cohort study to compare micafungin (at a dose of $100 \mathrm{mg}$ intravenously once per day) with caspofungin (loading dose of $70 \mathrm{mg}$ followed by $50 \mathrm{mg}$ once per day) for the treatment of febrile patients with persistent neutropenia who have hematological malignancies (prolonged neutropenia for $\geq 10$ days), thus being at higher risk to develop IFI. By establishing a minimum standard of five days of antifungal therapy, we hoped to increase the robustness of the conclusions drawn from the present cohort analysis.

\section{METHODS}

\section{Study design}

A retrospective cohort analysis involving patients with acute leukemia admitted to the Princess Margaret Cancer Centre (University Health Network, Toronto, Ontario) between April 2005 and November 2009 was conducted. All patients reviewed had received empirical antifungal therapy with either caspofungin or micafungin for persistent fever during a period of profound neutropenia. Caspofungin was the sole echinocandin used for persistent fever until June 2008, when micafungin was introduced as therapy for persistent fever in profoundly neutropenic patients. After that date, both agents were used for this indication. Patients were identified through the Department of Pharmacy dispensing records at the hospital and were enrolled in a 1:2 manner for micafungin versus caspofungin. Approval for data collection was obtained from the Research Ethics Board of the University Health Network (Toronto, Ontario).

\section{Patients}

Patients diagnosed with hematological malignancies housed in specific patient wards at the Princess Margaret Cancer Centre and who received caspofungin or micafungin were initially identified from pharmacy records. Thereafter, patients were only identified by their medical record number and data were retrieved from the electronic patient record. To be included in the present study, patients had to meet stipulated criteria: have a hematological malignancy; undergo induction, reinduction for failed remission or relapse, or consolidation chemotherapy, or have myelodysplastic syndrome (MDS) treated with azacytadine; be profoundly neutropenic, defined by $<0.5 \times 10^{9}$ neutrophils/L for a minimum of 10 days; and be treated with caspofungin or micafungin for a minimum of five days. Patients lacking these prespecified criteria were excluded.
The following information was collected: age; sex; length of hospital stay; duration of neutropenia (the first day the neutrophil count fell to $<0.5 \times 10^{9} / \mathrm{L}$ cells was considered to be day 1 of neutropenia, and the first day of neutrophil recovery $\geq 0.5 \times 10^{9} / \mathrm{L}$ cells was considered to be the end of the neutropenic period); primary diagnosis (acute myeloid leukemia, acute lymphoblastic leukemia [ALL], MDS, chronic myeloid leukemia); antifungal prophylaxis (drug and dose); type of chemotherapy (induction, reinduction or consolidation); chemotherapeutic agents; duration of neutropenia; start and end date of systemic antifungal therapy; computed tomography (CT) scan studies; treatment of baseline fungal infection; adverse events related to caspofungin and micafungin necessitating discontinuation of therapy; survival; and breakthrough fungal infections.

Data regarding the systemic empirical antifungal treatments were accrued as follows: the day on which either antifungal treatment was started was considered to be day 0 ; and assessments were performed again for each patient at days three to five of therapy, two weeks and four weeks. These assessments were documented as first, second and third reassessments, respectively.

\section{Treatment outcomes}

A composite end point was evaluated for each antifungal treatment course of micafungin and caspofungin, respectively. Antifungal treatment success reflected survival at seven days of antifungal treatment, successful treatment of baseline fungal infections, absence of adverse events due to the antifungal medication and the absence of breakthrough fungal infections. Fever, which is usually included as one of the variables of the composite end point, was not assessed due to the lack of consistent temperature records in the electronic patient record. Therefore, fever was not included as part of the composite end point.

Baseline fungal infection was defined as a fungal infection that was already identified on inclusion in the study or within $48 \mathrm{~h}$ of starting micafungin or caspofungin. Breakthrough fungal infection was defined as a fungal infection diagnosed $>48 \mathrm{~h}$ after starting antifungal treatment.

IFIs were defined according to the revised EORTC-MSG criteria (7). However, a possible IFI was determined on the basis of historical commentary of persistent fever unresponsive to appropriate antibacterial therapy with CT imaging findings consistent with pulmonary infection that did not meet the prespecified CT scan findings outlined in the EORTC-MSG criteria (7). Abnormal chest CT findings similar to those previously documented by others were considered to be consistent with IFI (8). The diagnosis of presumed fungal infection was made based on the presence of infectious symptoms of presumed fungal origin, regardless of whether antifungal prophylaxis was being administered, because appropriate antibacterial therapy was being used and there was no obvious focus of infection documented.

\section{Statistical analysis}

Demographic information for the treatment groups was assessed and compared using $\chi^{2}$ tests for categorical variables, Student's $t$ test for normally distributed continuous variables and rank-sum test for nonnormally distributed continuous variables. Overall success rates between the treatment groups were evaluated on the basis of the four parameters comprising the composite end point as stated above. Differences between the groups in overall success and each of the four end points were evaluated using $\chi^{2}$ testing. All analyses were performed using Stata version 12. A forest plot was generated using $\mathrm{R}$ version 3.1 .0 and the $95 \%$ CIs for the absolute difference between the treatment groups were calculated using a Cochran-Mantel Haenszel method (9).

\section{RESULTS}

The investigators initially identified 340 patients through retrospective chart review, who received echinocandin therapy with caspofungin or micafungin for fever and neutropenia during the study period. The disposition of the patients is illustrated in Figure 1. A total of 92 and 
118 patients were excluded from the caspofungin and micafungin groups, respectively. Reasons for exclusions were: duplicate entries; neutropenia for $<10$ days; receipt of the study drug for $<5$ days; or receipt of the study drug concomitantly with another agent. Patients who received interrupted courses of the study drug or received it while non-neutropenic, and those who were stem cell transplant recipients were also excluded. In addition, 58 patients were excluded from the micafungin group because they received prophylactic micafungin at a dose of $50 \mathrm{mg}$ per day.

The demographic characteristics of the patients analyzed in the study are summarized in Table 1 . The median ages of the patients were 47 and 54 years of age in the micafungin and caspofungin groups, respectively $(\mathrm{P}=0.02)$. There was no difference in the sex distribution between the groups, with $56 \%$ (27 of 48 ) and $61 \%$ (50 of 82 ) being male in the micafungin and the caspofungin groups, respectively $(\mathrm{P}=0.6)$. The median length of admission was similar in both groups ( 36 days in the micafungin and 39 days in the caspofungin patients; $\mathrm{P}=0.8$ ). Other baseline demographic characteristics were similar in both groups. The most common underlying diagnosis was acute myeloid leukemia (79\% [38 of 48] in the micafungin group and 83\% [68 of 82] in the caspofungin group). The remaining diagnoses were ALL, relapsed ALL and MDS. There were no significant differences in chemotherapeutic regimens used for the patients. Forty percent (19 of 48 ) of the micafungin group compared with $54 \%$ (44 of 82 ) of the caspofungin patients received induction regimens, while $42 \%$ (20 of 48 ) versus $30 \%$ ( 25 of 82 ) received reinduction chemotherapy. The remainder received consolidation treatment. The median duration of neutropenia was similar in in the micafungin and caspofungin groups ( 25 days and 29 days, respectively; $\mathrm{P}=0.7$ ).

Seventy-three percent (35 of 48) of the patients in the micafungin group received antifungal prophylaxis, in contrast to $57 \%$ (47 of 82 ) of the patients in the caspofungin group $(\mathrm{P}=0.08)$. Of the 48 patients who received antifungal prophylaxis in the micafungin group, 31 received fluconazole, while three received micafungin $50 \mathrm{mg}$ and one received voriconazole (Table 2). In the caspofungin group, 38 of 82 received fluconazole, one received caspofungin, five received voriconazole, one received itraconazole and two received amphotericin B. Caspofungin prophylaxis was provided to one patient in the caspofungin group for seven days before the development of neutropenia; the patient was then transitioned to empirical antifungal therapy. No baseline fungal infection was noted; however, the patient subsequently developed pulmonary CT findings indicative of a possible breakthrough infection and was considered to represent a failure. All patients received antibacterial agents for their febrile neutropenic episode, but no significant differences were noted between the two groups. In addition, there was

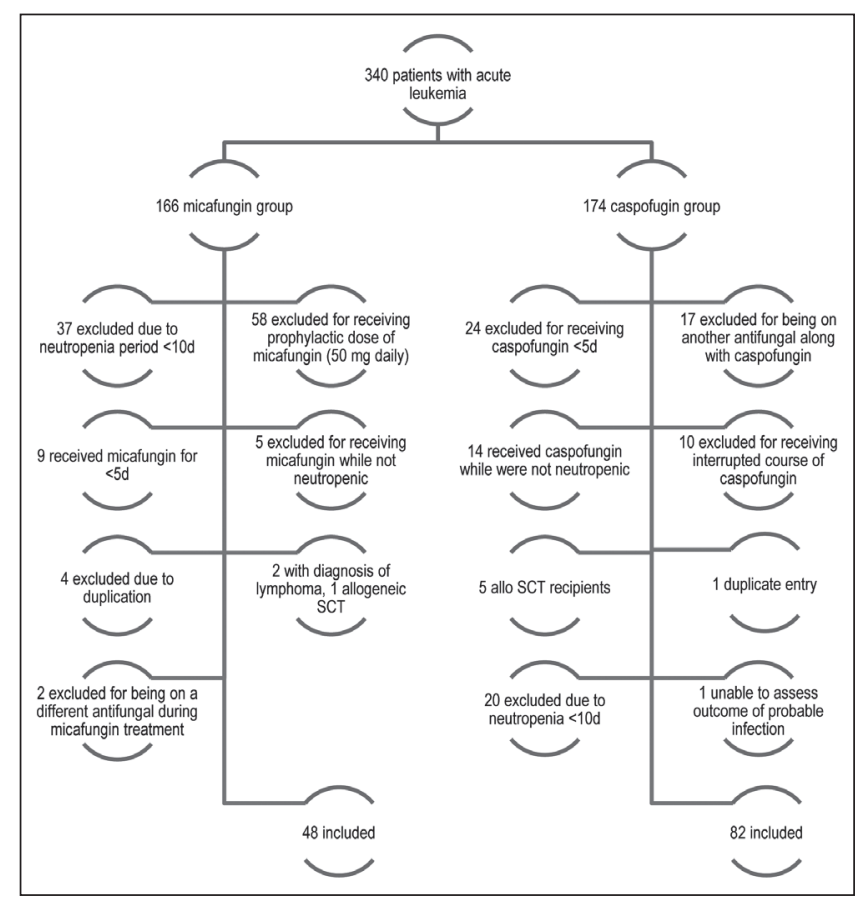

Figure 1) Consort diagram. d Days; SCT Stem cell transplant

a minority of patients in each group (nine in the micafungin group and 19 in the caspofungin group) who required previous antifungal therapy due to presumed fungal infection but were judged to have failed this therapy due to ongoing infectious symptoms and/or abnormal radiological findings and were, thus, placed on one of the study medications.

\section{Treatment outcomes}

The median duration of therapy for the micafungin and caspofungin groups was 16 days and 14.5 days, respectively $(\mathrm{P}=0.62)$ (Table 1$)$. As mentioned above, a successful treatment outcome was adjudicated based on four parameters: survival $\geq 7$ days after the discontinuation of antifungal therapy; treatment of baseline fungal infection; absence of breakthrough fungal infections; and absence of discontinuation of antifungal therapy due to adverse events. The treatment outcomes are summarized in Table 3. Both drugs performed similarly in all parameters except for survival. All patients in the micafungin group $(100 \%$ [48 of 48]) survived for a minimum of seven days after discontinuation

\section{TABLE 1}

\section{Demographic data}

\begin{tabular}{|c|c|c|c|}
\hline Variable & Micafungin $(n=48)$ & Caspofungin $(n=82)$ & $\mathbf{P}$ \\
\hline Mean age, years & 47 & 54 & 0.02 \\
\hline \multicolumn{4}{|l|}{ Sex } \\
\hline Male & $27(56)$ & $50(61)$ & 0.6 \\
\hline Duration of study drug, days, median (IQR) & $16(10-22.5)$ & $14.5(9-23)$ & \\
\hline Length of hospital stay, days, median & 36 & 39 & 0.8 \\
\hline Acute lymphoblastic leukemia & $9(19)$ & $12(15)$ & \\
\hline Other* & $1(2)$ & $2(2)$ & \\
\hline \multicolumn{4}{|l|}{ Chemotherapeutic regimen } \\
\hline Induction chemotherapy & $19(40)$ & $44(54)$ & 0.21 \\
\hline Reinduction chemotherapy & $20(42)$ & $25(30)$ & 0.2 \\
\hline Consolidation chemotherapy & $2(4)$ & $6(7)$ & 0.47 \\
\hline
\end{tabular}


TABLE 2

Antifungal prophylaxis

\begin{tabular}{lccc}
\hline Prophylaxis agent & $\begin{array}{c}\text { Micafungin } \\
(\mathbf{n}=\mathbf{4 8})\end{array}$ & $\begin{array}{c}\text { Caspofungin } \\
(\mathbf{n}=\mathbf{8 2})\end{array}$ & $\mathbf{P}$ \\
\hline Fluconazole & 31 & 38 & 0.34 \\
Micafungin* & 3 & 0 & 0.04 \\
Caspofungin & 0 & 1 & 0.39 \\
Amphotericin B & 0 & 2 & 0.22 \\
Voriconazole & 1 & 5 & 0.18 \\
Itraconazole & 0 & 1 & 0.39 \\
\hline
\end{tabular}

*Micafungin dose $50 \mathrm{mg}$ intravenously once per day

of micafungin compared with $89 \%$ (73 of 82 ) in the caspofungin group $(\mathrm{P}=0.02)$. As mentioned previously, all fungal infections were categorized according to the EORTC-MSG criteria as proven, probable and possible infections (7). The modified CT findings of the chest indicating a fungal infection, as described by others (8), were also adopted. Baseline fungal infections were defined as those diagnosed before or within $72 \mathrm{~h}$ after starting the study antifungal. There were no proven or probable baseline fungal infections among the micafungin patients. However, there were 19 patients (40\%) with possible IFIs at baseline and the remaining patients $(n=29)$ received micafungin as empirical treatment (60\%) for various symptoms (predominantly fever). In the caspofungin group, there were three $(4 \%)$ proven baseline infections $(\mathrm{P}=0.18)$. All were candidemias due to Candida albicans and all responded to the therapy. No patients in the caspofungin group had a probable fungal infection, while $56 \%$ (46 of 82 ) had a possible fungal infection ( $\mathrm{P}=0.07$ compared with micafungin) and the remaining $40 \%$ (33 of 82$)(P=0.03)$ received caspofungin as empirical antifungal treatment. Overall, baseline fungal infections (proven, probable and possible) were successfully treated in eight $(42 \%)$ patients in the micafungin treatment group compared with 26 (53\%) in the caspofungin group $(\mathrm{P}=0.42)$.

Breakthrough IFI was once again classified as per the EORTC-MSG criteria as proven, probable and possible infection (7). There were three proven breakthrough IFIs (three of 48 [6\%]) among the micafungin therapy patients (two candidemias cause by Candida parapsilosis and one biopsy-proven mucormycosis of the skin). No probable IFIs were observed in the micafungin-treated patients. However, eight $(17 \%)$ of 48 patients were classified as having possible IFIs with no identifiable pathogen noted. Thirty-seven (77\%) individuals had no breakthrough fungal infections. In contrast, none of the caspofungin-treated patients developed a proven $(\mathrm{P}=0.02)$ or probable IFI. A possible breakthrough IFI occurred in $11(13 \%)(\mathrm{P}=0.61)$; while 71 of the 82 patients $(86.6 \%)$ had no breakthrough IFI; this was not statistically significant compared with the micafungin group $(\mathrm{P}=0.16)$ (Table 3$)$.
No adverse events led to discontinuation of micafungin, whereas caspofungin therapy had to be stopped in one of the 82 patients $(1.2 \%)$ due to an adverse event manifested by elevated liver enzymes (aspartate aminotransferase $>5$ times the baseline value) $(\mathrm{P}=0.44)$ (Table 3).

Failure in any of the four parameters used to assess successful therapy resulted in determining failure of the course of empirical therapy for either antifungal agent. Thus, the overall success rate for micafungin was $60.4 \%$ (29 of 48), whereas for caspofungin it was 57.3\% (47 of 82) $(\mathrm{P}=0.729)$. The absolute differences between the micafungin and caspofungin patient groups (95\% CIs) with regard to each end point as well as overall success are presented in Figure 2.

\section{DISCUSSION}

Prompted by the high rate of IFIs in patients with acute leukemia and the subsequent morbidity and mortality of these infections in persistently febrile neutropenic patients (10), empirical antifungal therapy, aimed at targeting the most commonly encountered fungal pathogens (ie, Candida and Aspergillus species), is a commonly used strategy for managing persistently febrile neutropenic patients (1). One of the options for empirical antifungal therapy is the echinocandins. The echinocandins, caspofungin (3), micafungin (11) and anidulafungin (12), have demonstrated efficacy against Candida. Moreover, caspofungin, anidulafungin and micafungin have demonstrated efficacy in treating invasive aspergillosis alone or in combination with other antifungal agents (13-15). Although micafungin was shown to be superior to fluconazole when used for primary antifungal prophylaxis in stem cell transplant recipients, with reduction in the rate of breakthrough invasive Aspergillus infections in the micafungin arm (16), the optimal micafungin dose to be used in treating invasive aspergillosis is still unknown (15). Due to its lower wholesale noncontractual drug acquisition cost compared with caspofungin, micafungin offers a potential advantage.

However, while caspofungin has been approved as an empirical antifungal treatment for persistently febrile neutropenic cancer patients, the efficacy of micafungin for that indication is still unproven. This issue prompted Kubiak et al (6) to explore the use of micafungin as an empirical antifungal therapy in febrile neutropenic patients in a retrospective study. That study, however, had a number of limitations: specifically, a heterogeneous patient population with varying degrees and durations of neutropenia, as previously mentioned. We mandated a 10-day minimum duration of neutropenia for inclusion, which is the duration associated with the development of fungal infections (1). Moreover, a minimum duration of five days of treatment in each arm was required for inclusion to ensure proper assessment for efficacy and side effects. In the present retrospective study, we demonstrated that

\section{TABLE 3}

Treatment outcomes

\begin{tabular}{|c|c|c|c|}
\hline Treatment outcome & Micafungin $(n=48)$ & Caspofungin $(n=82)$ & $\mathbf{P}$ \\
\hline Survival $\geq 7$ days after stopping antifungal agent & $48(100)$ & $73(89)$ & 0.02 \\
\hline Successful treatment of baseline infections & $8 / 19(42)$ & $26 / 49(53)$ & 0.42 \\
\hline Proven baseline infection* & $0(0)$ & $3(3.7)$ & 0.18 \\
\hline No baseline infections & $29(60.4)$ & $33(40.2)$ & 0.03 \\
\hline Breakthrough fungal infections & $11(23)$ & $11(13.4)$ & 0.06 \\
\hline None & $37(77)$ & $71(87)$ & 0.16 \\
\hline Absence of adverse events ${ }^{\ddagger}$ & $48(100)$ & $81(99)$ & 0.44 \\
\hline \multicolumn{4}{|l|}{ Overall success } \\
\hline Yes & $29(60.4)$ & $47(57.3)$ & 0.73 \\
\hline No & $19(39.6)$ & $54(41.5)$ & \\
\hline
\end{tabular}

Data presented as $n(\%)$ unless otherwise indicated. *All proven baseline infections are due to Candida albicans candidemia; ${ }^{\dagger}$ Two cases of Candida parapsilosis and one case of mucormycosis of the skin; ${ }^{\ddagger}$ Defined as aspartate aminotransferase $>5$ times the upper limit of normal 
micafungin had a similar overall efficacy compared with caspofungin when used for treating persistently neutropenic patients with acute leukemia (overall efficacy of $60.4 \%$ for micafungin versus $57.3 \%$ for caspofungin). Efficacy was substantiated in a robust fashion by using four of five efficacy end points based on previously used criteria to evaluate and compare efficacy of antifungals for this purpose $(2,17,18)$. With regard to the evaluation parameters and a comparison of the treatment groups, proven baseline fungal infections were all candidemias, and occurred only in the caspofungin group, all of which were successfully treated. We also included possible baseline IFIs to enhance our results, demonstrating response rates of $42 \%$ in the micafungin group and $53 \%$ with caspofungin $(\mathrm{P}=0.42)$. This was performed in an attempt to provide additional information about the patients' disposition. Breakthrough infections occurred only in the micafungin group; two were $\mathrm{C}$ parapsilosis candidemias, an expected breakthrough infection considering the welldocumented inherent increased minimum inhibitory concentrations of C parapsilosis species to echinocandins (19). The third was due to biopsy-proven mucormycosis of the skin - once more, a weakness in the antifungal coverage of the echinocandins (19). Although our data demonstrate similar efficacy of micafungin compared with caspofungin when used to treat persistently neutropenic patients, one may question the robustness of this conclusion given the exclusion of fever as an end point. Nonetheless, the utility of including fever as a primary composite end point has been questioned by investigators, primarily due to the lack of specificity of fever as a sign of occult fungal infection, particularly in the studied population $(20,21)$ and its lack of accuracy as a harbinger for IFI (22,23).

We acknowledge that the present study had some other limitations. The retrospective nature limited the ability to control for all the potential confounding factors, although the cohort design lessened this issue somewhat. There were also few proven baseline fungal infections and all occurred in the caspofungin group. In addition, there were no proven mold infections among the included patients, which makes drawing firm conclusions regarding the efficacy of micafungin to treat mold infections impossible. Furthermore, surrogate markers for invasive mold infection, such as galactomannan assays, were not available at the institution at the time of patients' hospitalizations, which may partially explain the high number of possible IFIs and the absence of probable IFIs. The lack of adherence to the standardized EORTC-MSG radiological criteria in our study may be viewed as another limitation. However, strict adherence to these radiological criteria may result in unnecessary exclusion of patients from trials that assess efficacy of antifungal therapies in treating IFIs in neutropenic populations, as previously suggested (8). To substantiate false identification of patients with bacterial respiratory tract infections as possible invasive pulmonary

\section{REFERENCES}

1. Freifeld AG, Bow EJ, Sepkowitz KA, et al. Clinical practice guideline for the use of antimicrobial agents in neutropenic patients with cancer: 2010 update by the Infectious Diseases Society of America. Clin Infect Dis 2011;52:e56-93.

2. Walsh TJ, Teppler H, Donowitz GR, et al. Caspofungin versus liposomal amphotericin B for empirical antifungal therapy in patients with persistent fever and neutropenia. N Engl J Med 2004;351:1391-402.

3. Mora-Duarte J, Betts R, Rotstein C, et al. Comparison of caspofungin and amphotericin B for invasive candidiasis. N Engl J Med 2002;347:2020-9.

4. Pappas PG, Kauffman CA, Andes D, et al. Clinical practice guidelines for the management of candidiasis: 2009 update by the Infectious Diseases Society of America. Clin Infect Dis 2009;48:503-35.

5. Tomblyn M, Chiller T, Einsele H, et al. Guidelines for preventing infectious complications among hematopoietic cell transplantation recipients: A global perspective. Biol Blood Marrow Transplant 2009;15:1143-238.

6. Kubiak DW, Bryar JM, McDonnell AM, et al. Evaluation of caspofungin or micafungin as empiric antifungal therapy in adult patients with persistent febrile neutropenia: A retrospective, observational, sequential cohort analysis. Clin Ther 2010;32:637-48.

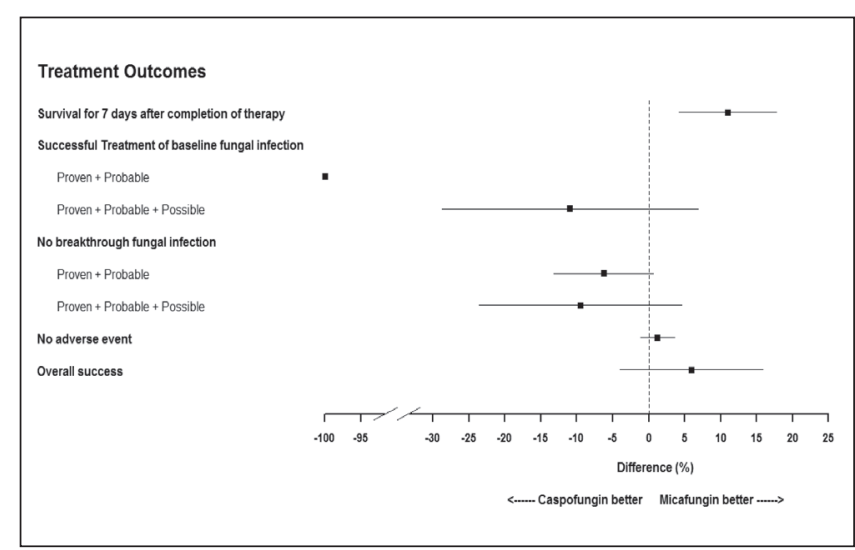

Figure 2) Treatment outcomes reflecting absolute difference between micafungin and caspofungin groups with 95\% CIs (micafungin result caspofungin result)

fungal infections, all patients with possible or probable infections based on abnormal radiological findings had negative sputum or bronchoalveolar lavage bacterial cultures at the time of inclusion.

\section{CONCLUSION}

Micafungin demonstrated similar efficacy to caspofungin when used to empirically treat febrile neutropenic patients with hematological malignancies, as shown by the composite end point. Our study also substantiates the safety profile of micafungin at a dose of $100 \mathrm{mg}$. A prospective, randomized clinical trial comparing caspofungin and micafungin for treatment of persistently febrile neutropenic patients with focus on outcomes with mold infections is warranted.

CONFLICT OF INTERESTS: Coleman Rotstein MD has been paid consultant fees and/or speaker honoraria from Astellas Pharma Canada Inc, Merck Canada Inc, Cubist Pharmaceuticals Canada Inc and Pfizer Canada as well as received research grant support from Astellas Pharma Canada Inc, Merck Canada Inc and Pfizer Canada. Shahid Husain MD has received research grant support from Pfizer Canada. The remaining authors report no conflicts of interest related to this article.

ACKNOWLEDGEMENTS: The authors acknowledge the contribution of Dr Yanhong Li in the analysis of the data in this article. This research study was supported in part by a grant from Astellas Pharma Canada Inc.

7. De Pauw B, Walsh TJ, Donnelly JP, et al. Revised definitions of invasive fungal disease from the European Organization for Research and Treatment of Cancer/Invasive Fungal Infections Cooperative Group and the National Institute of Allergy and Infectious Diseases Mycoses Study Group (EORTC/MSG) Consensus Group. Clin Infect Dis 2008;46:1813-21.

8. Nucci M, Nouer SA, Grazziutti M, Kumar NS, Barlogie B, Anaissie E. Probable invasive aspergillosis without prespecified radiologic findings: Proposal for inclusion of a new category of aspergillosis and implications for studying novel therapies. Clin Infect Dis 2010;51:1273-80.

9. Koch G, Carr G, Amara I, Stokes M, Urynink T. Categorical data analysis. In: Berry D, ed. Statistical Methodology in Pharmaceutical Sciences. New York: Marcel Dekker, 1990:414-21.

10. Neofytos D, Lu K, Hatfield-Seung A, et al. Epidemiology, outcomes, and risk factors of invasive fungal infections in adult patients with acute myelogenous leukemia after induction chemotherapy. Diagn Microbiol Infect Dis 2013;75:144-9.

11. Pappas PG, Rotstein CM, Betts RF, et al. Micafungin versus caspofungin for treatment of candidemia and other forms of invasive candidiasis. Clin Infect Dis 2007;45:883-93.

12. Reboli AC, Rotstein C, Pappas PG, et al. Anidulafungin versus fluconazole for invasive candidiasis. N Engl J Med 2007;356:2472-82. 
13. Maertens J, Raad I, Petrikkos G, et al. Efficacy and safety of caspofungin for treatment of invasive aspergillosis in patients refractory to or intolerant of conventional antifungal therapy. Clin Infect Dis 2004;39:1563-71.

14. Marr KA, Schlamm H, Rottinghaus ST, et al. A randomized, doubleblind study of combination antifungal therapy with voriconazole and anidulafungin versus voriconazole monotherapy for primary treatment of invasive aspergillosis. Astract LB 2812. 22nd European Congress of Clinical Microbiology and Infectious Diseases (ECCMID). London, March 31 to April 3, 2012.

15. Denning DW, Marr KA, Lau WM, et al. Micafungin (FK463), alone or in combination with other systemic antifungal agents, for the treatment of acute invasive aspergillosis. J Infect 2006;53:337-49.

16. van Burik JA, Ratanatharathorn V, Stepan DE, et al. Micafungin versus fluconazole for prophylaxis against invasive fungal infections during neutropenia in patients undergoing hematopoietic stem cell transplantation. Clin Infect Dis 2004;39:1407-16.

17. Walsh TJ, Pappas P, Winston DJ, et al. Voriconazole compared with liposomal amphotericin B for empirical antifungal therapy in patients with neutropenia and persistent fever. $\mathrm{N}$ Engl J Med 2002;346:225-34.

18. Walsh TJ, Finberg RW, Arndt C, et al. Liposomal amphotericin B for empirical therapy in patients with persistent fever and neutropenia. National Institute of Allergy and Infectious Diseases Mycoses Study Group. N Engl J Med 1999;340:764-71.

19. Ashley ES, Lewis R, Lewis JS, Martin C, Andes D. Pharmacology of systemic antifungal agents. Clin Infect Dis 2006;43:S28-29.

20. Bennett JE, Powers J, Walsh T, et al. Forum report: Issues in clinical trials of empirical antifungal therapy in treating febrile neutropenic patients. Clin Infect Dis 2003;36(Suppl 3):S117-22.

21. de Pauw BE, Sable CA, Walsh TJ, et al. Impact of alternate definitions of fever resolution on the composite endpoint in clinical trials of empirical antifungal therapy for neutropenic patients with persistent fever: Analysis of results from the Caspofungin Empirical Therapy Study. Transpl Infect Dis 2006;8:31-7.

22. Morrissey CO, Chen SC, Sorrell TC, et al. Galactomannan and PCR versus culture and histology for directing use of antifungal treatment for invasive aspergillosis in high-risk haematology patients: A randomised controlled trial. Lancet Infect Dis 2013;13:519-28.

23. Maertens J, Theunissen K, Verhoef G, et al. Galactomannan and computed tomography-based preemptive antifungal therapy in neutropenic patients at high risk for invasive fungal infection: A prospective feasibility study. Clin Infect Dis 2005;41:1242-50. 


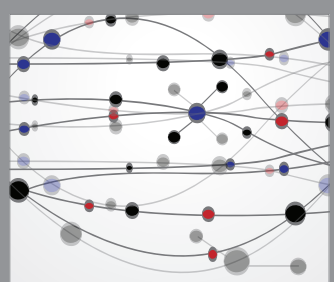

The Scientific World Journal
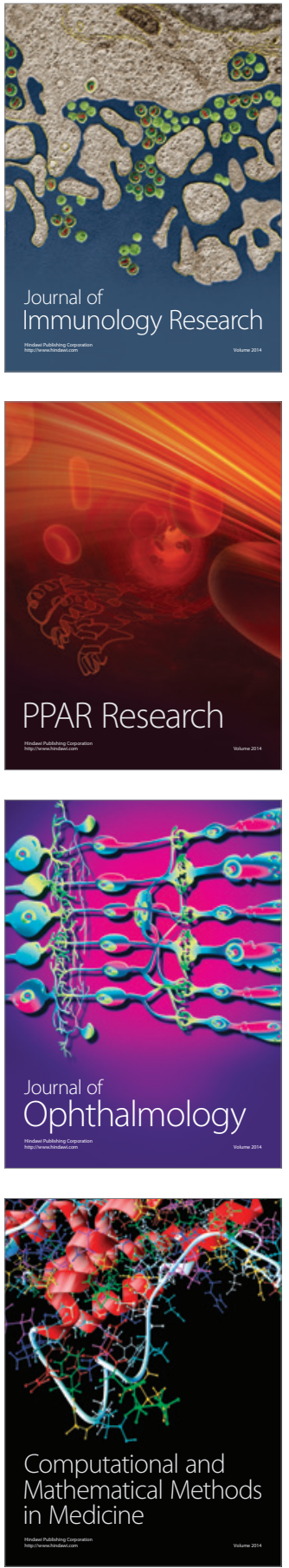

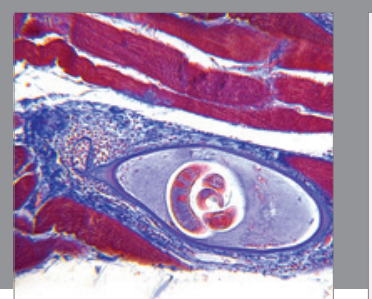

Gastroenterology Research and Practice

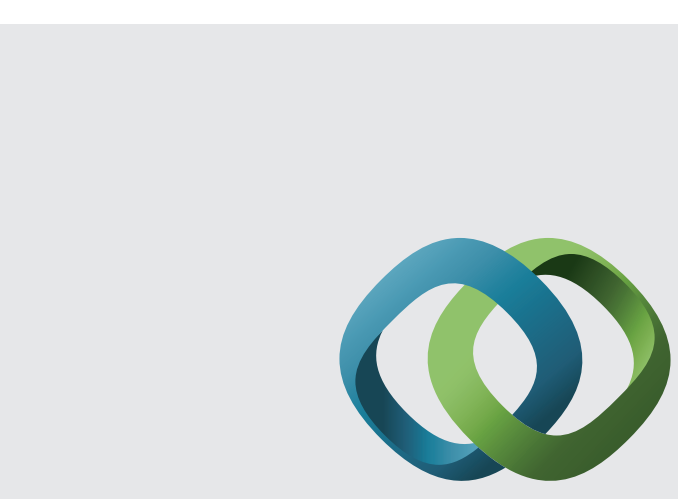

\section{Hindawi}

Submit your manuscripts at

http://www.hindawi.com
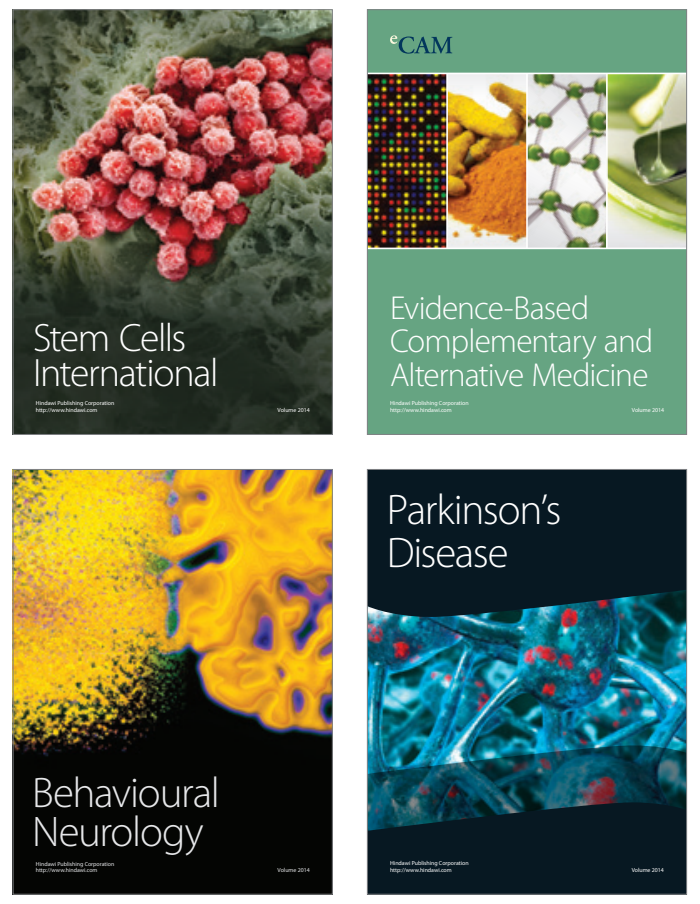
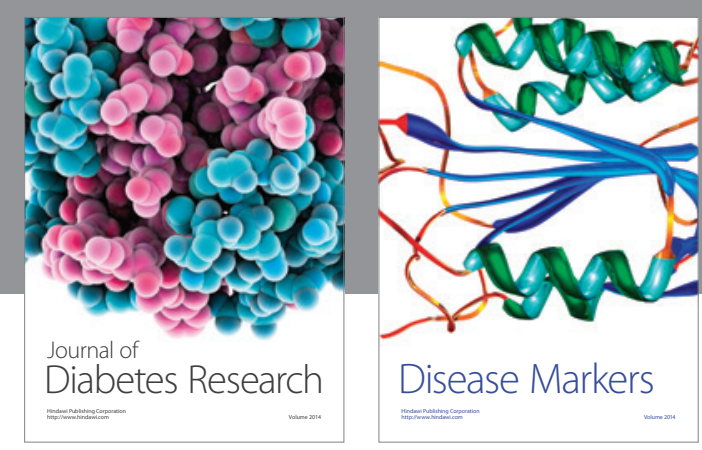

Disease Markers
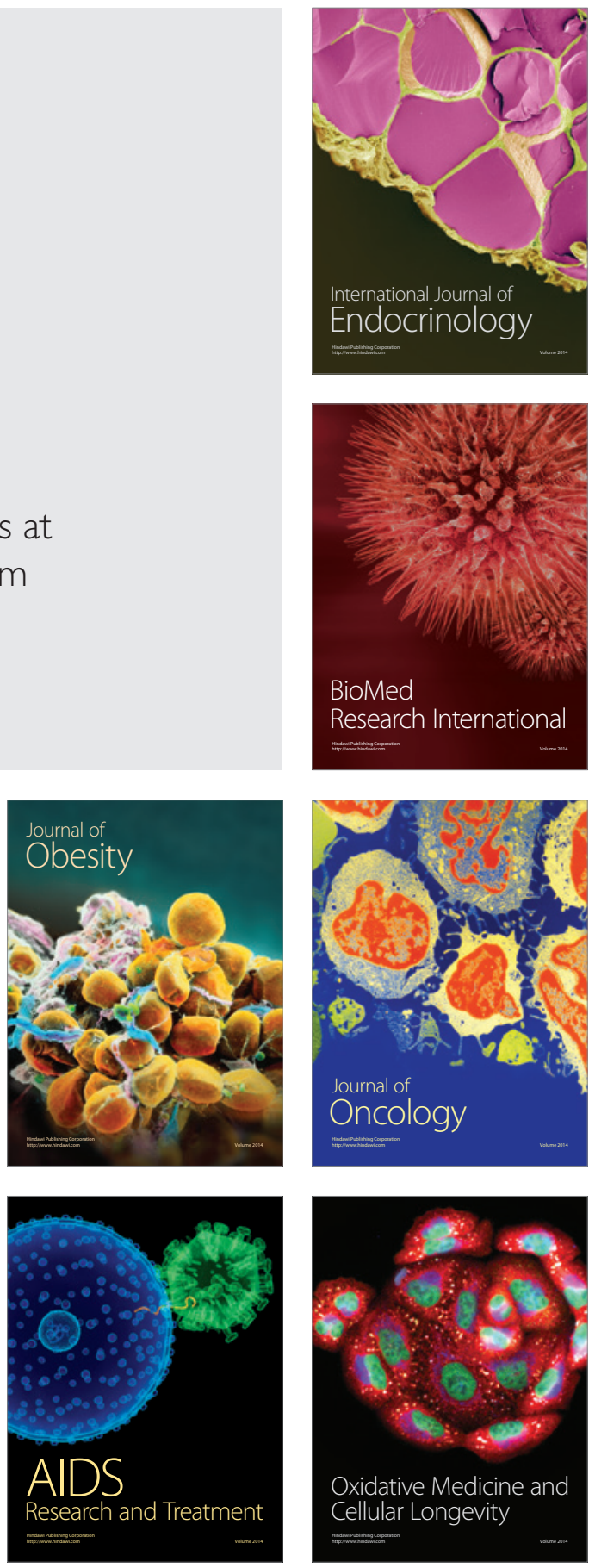